\title{
Edge-superconnectivity of semiregular cages with odd girth
}

\author{
Camino Balbuena and Julián Salas \\ Universitat Politc̀nica de Catalunya \\ Barcelona \\ Diego González-Moreno \\ Universidad Nacional Autónoma de México \\ México
}

\begin{abstract}
A graph is said to be edge-superconnected if each minimum edge-cut consists of all the edges incident with some vertex of minimum degree. A graph $G$ is said to be a $\{d, d+1\}$ semiregular graph if all its vertices have degree either $d$ or $d+1$. A smallest $\{d, d+1\}$-semiregular graph $G$ with girth $g$ is said to be a $(\{d, d+1\} ; g)$-cage. We show that every $(\{d, d+1\} ; g)$-cage with odd girth $g$ is edge-superconnected.
\end{abstract}

\section{Introduction}

We only consider undirected simple graphs without loops or multiple edges. Unless otherwise stated, we follow [9] for basic terminology and definitions. Let $G$ stand for a graph with vertex set $V=V(G)$ and edge set $E=$ $E(G)$. The distance $d_{G}(u, v)=d(u, v)$ between two vertices of the graph $G$ is the length of a shortest path between $u$ and $v$, and the diameter of $G$ denoted by $\operatorname{diam}(G)$ is the maximum distance between any pair of vertices; when $G$ is not connected, then $\operatorname{diam}(G)=+\infty$. For $w \in V$ and $S \subset V, d(w, S)=d_{G}(w, S)=\min \{d(w, s): s \in S\}$ denotes the distance between $w$ and $S$. For every $S \subset V$ and every nonnegative integer $r \geq 0$, $N_{r}(S)=\{w \in V: d(w, S)=r\}$ denotes the neighborhood of $S$ at distance $r$. Thus the set of vertices adjacent to a vertex $v$ is $N(v)=N_{1}(\{v\})$, and 
Edge-superconnectivity

of semiregular cages with odd girth

C. Balbuena et al.

the degree of a vertex $v$ in $G$ is $\operatorname{deg}_{G}(v)=\operatorname{deg}(v)=|N(v)|$, whereas the minimum degree $\delta=\delta(G)$ is the minimum degree over all vertices of $G$. A graph is called $r$-regular if every vertex of the graph has degree $r$.

A graph $G$ is called connected if every pair of vertices is joined by a path. An edge-cut in a graph $G$ is a set $W$ of edges of $G$ such that $G-W$ is disconnected. A graph is $k$-edge-connected if every edge-cut contains at least $k$ edges. If $W$ is a minimal edge-cut of a connected graph $G$, then necessarily, $G-W$ contains exactly two components. The edge-connectivity $\lambda=\lambda(G)$ of a graph $G$ is the minimum cardinality of an edge-cut of $G$. A classic result is $\lambda \leq \delta$ for every graph $G$. A graph is maximally edgeconnected if $\lambda=\delta$.

One might be interested in more refined indices of reliability. Even two graphs with the same edge-connectivity $\lambda$ may be considered to have different reliabilities. As a more refined index than the edge-connectivity, edge-superconnectivity is proposed in $[6,7]$. A subset of edges $W$ is called trivial if it contains the set of edges incident with some vertex of the graph. Clearly, if $|W| \leq \delta-1$, then $W$ is nontrivial. A graph is said to be edgesuperconnected if $\lambda=\delta$ and every minimum edge-cut is trivial.

The degree set $D$ of a graph $G$ is the set of distinct degrees of the vertices of $G$. The girth $g(G)$ is the length of a shortest cycle in $G$. A $(D ; g)$-graph is a graph having degree set $D$ and girth $g$. Let $n(D ; g)$ denote the least order of a $(D ; g)$-graph. Then a $(D ; g)$-graph with order $n(D ; g)$ is called a $(D ; g)$ cage. If $D=\{r\}$ then a $(D ; g)$-cage is a $(r ; g)$-cage. When $D=\{r, r+1\}$, we refer to $(D ; g)$-cages as semiregular cages.

The existence of $(r ; g)$-cages was proved by Erdös and Sachs [10] in the decade of the 60's, and using this result Chartrand et al. [8] proved the existence of $(D ; g)$-cages. Some of the structural properties of $(r ; g)$ cages that have been studied are the vertex and the edge connectivity; concerning this problem $\mathrm{Fu}$, Huang and Rodger [11] conjectured that every $(r ; g)$-cage is $r$-connected, and they proved the statement for $r=3$. Other contributions supporting this conjecture can be seen in $[15,16,17,20]$. Moreover, some structural properties of $(r ; g)$-cages have been extended for $(D ; g)$-cages, for example the monotonicity of the order with respect to the girth (see Theorem 1) and the upper bound for the diameter (see Theorem 2 ). The edge-superconnectivity of cages was established in [18, 19]. For semiregular cages, it has been proved in [3] that they are maximally edge connected. The main objective of this work is to prove that every $(\{d, d+$ 
Edge-superconnectivity

of semiregular cages with odd girth

C. Balbuena et al.

$1\} ; g)$-cage with odd girth $g \geq 5$ is edge-superconnected. With this aim we need the following two results.

Theorem 1 [4] Let $g_{1}, g_{2}$ be two integers such that $3 \leq g_{1}<g_{2}$. Then $n\left(\{d, d+1\} ; g_{1}\right)<n\left(\{d, d+1\} ; g_{2}\right)$.

Theorem $2[5]$ The diameter of a $(\{d, d+1\} ; g)$-cage is at most $g$.

\section{Main theorem}

In order to study the edge-superconnectivity of a graph in terms of its diameter and its girth, the following results were established $[1,2,13]$.

Proposition 3 Let $G=(V, E)$ be a connected graph with minimum degree $\delta \geq 2$ and girth $g$. Let $W \subset E$ be a minimum nontrivial edge-cut, let $H_{i}$ be a component of $G-W$, and let $W_{i} \subset V\left(H_{i}\right)$ be the set of vertices of $H_{i}$ which are incident with some edge in $W, i=0,1$. Then there exists some vertex $x_{i} \in V\left(H_{i}\right)$ such that

(a) $[1,13] d\left(x_{i}, W_{i}\right) \geq\lfloor(g-1) / 2\rfloor$, if $\left|W_{i}\right| \leq \delta-1$.

(b) [2] $d\left(x_{i}, W_{i}\right) \geq\lceil(g-3) / 2\rceil$, if $|W| \leq \xi-1$, where $\xi=\min \{\operatorname{deg}(u)+$ $\operatorname{deg}(v)-2: u v \in E\}$ is the minimum edge-degree of $G$.

For every minimum edge-cut $W$ of $G$ such that $H_{0}, H_{1}$ are the two components of $G-W$, we will write henceforth $W=\left[W_{0}, W_{1}\right]$ with $W_{0} \subset$ $V\left(H_{0}\right)$ and $W_{1} \subset V\left(H_{1}\right)$ containing all endvertices of the edges in $W$. Note that $\left|W_{i}\right| \leq|W|, i=0,1$. From now on, let

$$
\mu_{i}=\max \left\{d\left(x, W_{i}\right): x \in V\left(H_{i}\right)\right\}, \quad i=0,1 .
$$

When $W$ is nontrivial and $|W| \leq \xi-1$, it follows from Proposition 3 that $\mu_{i} \geq\lceil(g-3) / 2\rceil$. Likewise, $\mu_{0}$ and $\mu_{1}$ satisfy some other basic properties shown in next lemma.

Lemma 4 Let $G=(V, E)$ be a connected graph with minimum degree $\delta \geq 3$ and odd girth $g \geq 5$. Let $W=\left[W_{0}, W_{1}\right] \subset E$ be a minimum nontrivial edgecut with cardinality $|W| \leq \delta$. Let $G-W=H_{0} \cup H_{1}$, where $W_{i} \subset V\left(H_{i}\right)$. If $\mu_{i}=(g-3) / 2$ the following statements hold: 
Edge-superconnectivity

of semiregular cages with odd girth

C. Balbuena et al.

(i) $\left|W_{i}\right|=|W|=\delta$, and every $a \in W_{i}$ is incident to a unique edge of $W$.

(ii) Every vertex $z \in V\left(H_{i}\right)$ such that $d\left(z, W_{i}\right)=\mu_{i}$ has $\operatorname{deg}(z)=\delta$.

(iii) For every $a \in W_{i}$ there exists a vertex $x \in V\left(H_{i}\right)$ such that $d\left(x, W_{i}\right)=$ $d(x, a)=\mu_{i}$ and $N_{(g-3) / 2}(x) \cap W_{i}=\{a\}$. Further, $N(x)$ can be labeled as $\left\{u_{1}, u_{2}, \ldots, u_{\delta}\right\}$, and $W_{i}$ can be labeled as $\left\{a_{1}, a_{2}, \ldots, a_{\delta}\right\}$, where $a_{1}=a$, so that $N_{(g-5) / 2}\left(u_{1}\right) \cap W_{i}=\left\{a_{1}\right\}$ and $N_{(g-3) / 2}\left(u_{k}\right) \cap W_{i}=\left\{a_{k}\right\}$ for every $k>1$. Consequently $\left|\left[N_{(g-3) / 2}(x) \cap W_{i}, W_{i+1}\right]\right|=1$ and $\left|\left[N_{(g-3) / 2}\left(u_{k}\right) \cap W_{i}, W_{i+1}\right]\right|=1$ (with subscripts taken mod 2). See Figure 2.

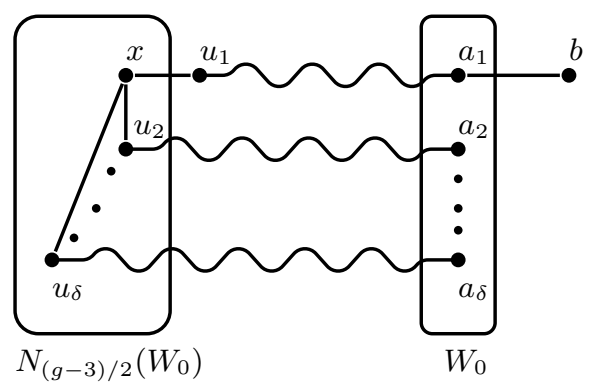

Figure 1: Lemma 4.

Proof: (i) Since $\mu_{i}=(g-3) / 2, d\left(x, W_{i}\right) \leq \mu_{i}=(g-3) / 2<(g-1) / 2$ for all $x \in V\left(H_{i}\right)$. Hence from Proposition 3 (a), it follows that $\left|W_{i}\right| \geq \delta$, yielding $\left|W_{i}\right|=\delta$ because $\left|W_{i}\right| \leq|W| \leq \delta$. Observe that $\delta=\left|W_{i}\right|=|W|$ means that $\left|N(a) \cap W_{i+1}\right|=1$ for each vertex $a \in W_{i}$ (taking the subscripts $\bmod 2$ ).

(ii) First observe that $\mu_{i}=(g-3) / 2 \geq 1$ since $g \geq 5$. Let us define the following partition of $N(v)$ for all $v \in V\left(H_{i}\right)$

$$
\begin{aligned}
& S^{-}(v)= \begin{cases}\left\{z \in N(v): d\left(z, W_{i}\right)=d\left(v, W_{i}\right)-1\right\} & \text { if } v \notin W_{i} ; \\
W_{i+1} \cap N(v) & \text { if } v \in W_{i} .\end{cases} \\
& S^{+}(v)=\left\{z \in N(v): d\left(z, W_{i}\right)=d\left(v, W_{i}\right)+1\right\} \\
& S^{=}(v)=\left\{z \in N(v): d\left(z, W_{i}\right)=d\left(v, W_{i}\right)\right\} .
\end{aligned}
$$


Edge-superconnectivity

of semiregular cages with odd girth

C. Balbuena et al.

Let $z$ be a vertex of $H_{i}$ such that $d\left(z, W_{i}\right)=\mu_{i}=(g-3) / 2$. Then we have

$$
\begin{aligned}
N(z) & =S^{=}(z) \cup S^{-}(z) ; \\
\left|N_{(g-3) / 2}\left(S^{=}(z)\right) \cap W_{i}\right| & \geq\left|S^{=}(z)\right| ; \\
\left|N_{(g-5) / 2}\left(S^{-}(z)\right) \cap W_{i}\right| & \geq\left|S^{-}(z)\right| ; \\
\left.N_{(g-3) / 2}\left(S^{=}(z)\right) \cap N_{(g-5) / 2}\left(S^{-}(z)\right)\right) & =\emptyset
\end{aligned}
$$

because otherwise cycles of length less than the girth $g$ appear. Since

$$
\begin{aligned}
\delta \leq \operatorname{deg}(z) & =\left|S^{=}(z)\right|+\left|S^{-}(z)\right| \\
& \leq\left|N_{(g-3) / 2}\left(S^{=}(z)\right) \cap W_{i}\right|+\left|N_{(g-5) / 2}\left(S^{-}(z)\right) \cap W_{i}\right| \\
& \leq\left|W_{i}\right|=\delta
\end{aligned}
$$

it follows that $\delta=\operatorname{deg}(z)$. Therefore item (ii) holds.

(iii) First let us prove that there exists an edge $z z^{\prime}$ such that $d\left(z, W_{i}\right)=$ $d\left(z^{\prime}, W_{i}\right)=(g-3) / 2$. Otherwise, $S^{=}(z)=\emptyset$ for all $z$ with $d\left(z, W_{i}\right)=$ $(g-3) / 2$. This implies that for all $u \in N(z), u \in S^{-}(z)$ and $S^{=}\left(S^{+}(u)\right)=\emptyset$. Further, $\left|N_{(g-5) / 2}(u) \cap W_{i}\right|=1$ for all $u \in N(z)$, because $\delta=\left|W_{i}\right|=$

$\sum_{u \in N(z)}\left|N_{(g-5) / 2}(u) \cap W_{i}\right| \geq \delta$. Hence $\left|S^{-}(u)\right|=1$, and so $\left|S^{+}(u)\right|+\left|S^{=}(u)\right|=$ $\operatorname{deg}(u)-1 \geq \delta-1 \geq 2$. Suppose that $\left|S^{=}(u)\right| \geq 1$ for some $u \in N(z)$. Then as $N_{(g-3) / 2}(z) \cap W_{i}$ and $N_{(g-5) / 2}\left(S^{=}(u)\right) \cap W_{i}$ are two vertex disjoint sets we have $|W| \geq\left|N_{(g-3) / 2}(z) \cap W_{i}\right|+\left|N_{(g-5) / 2}\left(S^{=}(u)\right) \cap W_{i}\right| \geq \delta+1$ which is a contradiction because $|W|=\delta$. Then we must assume that for all $u \in N(z),\left|S^{+}(u)\right|=\operatorname{deg}(u)-1 \geq \delta-1 \geq 2$. Let $t \in S^{+}(u)-z$, according to our first assumption $S^{=}(t)=\emptyset$ meaning that $N(t)=S^{-}(t)$. Since $t$ has the same behavior as $z$ we have $W_{i}=N_{(g-3) / 2}\left(S^{-}(z)\right)=N_{(g-3) / 2}\left(S^{-}(t)\right)$, and as $2<\delta \leq \operatorname{deg}(z)=\operatorname{deg}(t)$, there exist cycles through $\{z, u, t, w\}$ for some $w \in W_{i}$ of length less than $g$ which is a contradiction.

Hence we may assume that there exists an edge $z z^{\prime}$ such that $d\left(z, W_{i}\right)=$ $d\left(z^{\prime}, W_{i}\right)=(g-3) / 2$. Since $N_{(g-5) / 2}\left(S^{-}(z)\right) \cap W_{i}, N_{(g-5) / 2}\left(S^{-}\left(z^{\prime}\right)\right) \cap W_{i}$ and $N_{(g-3) / 2}\left(S^{=}\left(z^{\prime}\right)-z\right) \cap W_{i}$ are three pairwise disjoint sets because $g \geq 5$, and taking into account (1) we have

$$
\begin{aligned}
\delta=|W| \geq & \left|N_{(g-5) / 2}\left(S^{-}(z)\right) \cap W_{i}\right|+\left|N_{(g-5) / 2}\left(S^{-}\left(z^{\prime}\right)\right) \cap W_{i}\right| \\
& +\left|N_{(g-3) / 2}\left(S^{=}\left(z^{\prime}\right)-z\right) \cap W_{i}\right| \\
\geq & \left|S^{-}(z)\right|+\left|S^{-}\left(z^{\prime}\right)\right|+\left|S^{=}\left(z^{\prime}\right)-z\right| \\
= & \operatorname{deg}(z)-1+\left|S^{-}(z)\right| \geq \delta .
\end{aligned}
$$


Edge-superconnectivity

of semiregular cages with odd girth

C. Balbuena et al.

Therefore, all inequalities become equalities, i.e., $\left|S^{-}(z)\right|=1=\mid N_{(g-5) / 2}\left(S^{-}(z)\right) \cap$ $W_{i} \mid$. So $S^{-}(z)=\left\{z_{1}\right\}$ and $N(z)-z_{1}=S^{=}(z)$ yielding a partition of $W_{i}$ :

$$
W_{i}=\left(N_{(g-5) / 2}\left(z_{1}\right) \cap W_{i}\right) \cup\left(\cup_{z^{\prime} \in N(z)-z_{1}} N_{(g-3) / 2}\left(z^{\prime}\right) \cap W_{i}\right),
$$

because for all $z^{\prime} \in N(z)-z_{1}$ the sets $N_{(g-3) / 2}\left(z^{\prime}\right) \cap W_{i}$ and the set $N_{(g-5) / 2}\left(z_{1}\right) \cap W_{i}$ are mutually disjoint. Thus, $\left|N_{(g-3) / 2}\left(z^{\prime}\right) \cap W_{i}\right|=1$ for all $z^{\prime} \in N(z)-z_{1}$. Therefore, for every vertex $a \in W_{i}$ there exists a vertex $x \in\left(N(z)-z_{1}\right) \cup\{z\}$ such that $d\left(x, W_{i}\right)=d(x, a)=(g-3) / 2$ and $N_{(g-3) / 2}(x) \cap W_{i}=\{a\}$. Furthermore, since every vertex $z^{\prime} \in N(z)-z_{1}$ has the same behavior as $z, N(x)$ can be labeled as $\left\{u_{1}, u_{2}, \ldots, u_{\delta}\right\}$, and $W_{i}$ can be labeled as $\left\{a_{1}, a_{2}, \ldots, a_{\delta}\right\}$, where $a_{1}=a$, so that $N_{(g-5) / 2}\left(u_{1}\right) \cap W_{i}=$ $\left\{a_{1}\right\}$ and $N_{(g-3) / 2}\left(u_{k}\right) \cap W_{i}=\left\{a_{k}\right\}$ for every $k>1$. Finally, using (i) we obtain $\left|\left[N_{(g-3) / 2}(x) \cap W_{i}, W_{i+1}\right]\right|=1$ and $\left|\left[N_{(g-3) / 2}\left(u_{k}\right) \cap W_{i}, W_{i+1}\right]\right|=1$, which finishes the proof.

A semiregular cage is known to be maximally edge-connected [3]. Now, we are ready to prove that semiregular cages with odd girth are edgesuperconnected. As will be seen, Hall's Theorem is a key point of this study. Recall that if $S$ is a set of vertices in a graph $G$, the set of all neighbors of the vertices in $S$ is denoted by $N(S)$.

Theorem 5 ([12] Hall's Theorem) A bipartite graph with bipartition $\left(X_{1}, X_{2}\right)$ has a matching which covers every vertex in $X_{1}$ if and only if

$$
|N(S)| \geq|S| \text { for all } S \subset X_{1} .
$$

Using Hall's Theorem Jiang [14] proved the following result.

Lemma 6 [14] Let $G$ be a bipartite graph with bipartition $\left(X_{1}, X_{2}\right)$ where $\left|X_{1}\right|=\left|X_{2}\right|=r$. If $G$ contains at least $r^{2}-r+1$ edges, then $G$ contains a perfect matching.

The following lemma is an stronger version of Lemma 6, which is also proved using Hall's Theorem.

Lemma 7 Let $\mathcal{B}$ be a bipartite graph with bipartition $\left(X_{1}, X_{2}\right)$ where $\left|X_{1}\right|=$ $\left|X_{2}\right|=r$. If $\delta(\mathcal{B}) \geq 1$ and $|E(\mathcal{B})| \geq r^{2}-r$, then $\mathcal{B}$ contains a perfect matching. 
Edge-superconnectivity

of semiregular cages with odd girth

C. Balbuena et al.

Proof: Let $\mathcal{B}=\left(X_{1}, X_{2}\right)$ be a bipartite graph with $\left|X_{1}\right|=\left|X_{2}\right|=r$, $\delta(\mathcal{B}) \geq 1$ and $|E(\mathcal{B})| \geq r^{2}-r$. We shall apply Hall's Theorem to prove the lemma; we shall show that for a subset $S \subset X_{1},|N(S)| \geq|S|$. Notice that if $|S|=1$, then $|N(S)| \geq 1=|S|$ because $\delta(\mathcal{B}) \geq 1$; and if $S=X_{1}$, $N(S)=X_{2}$ because $\delta(\mathcal{B}) \geq 1$ implies that each vertex $u \in X_{2}$ must have a neighbor in $S$, hence $|S|=|N(S)|$.

Therefore we continue the proof reasoning by contradiction and so assuming that $1 \leq|N(S)|<|S|=t \leq r-1$. Then the number of edges in $\mathcal{B}$ is at most

$$
|E(\mathcal{B})|=|[S, N(S)]|+\left|\left[X_{1} \backslash S, X_{2}\right]\right| \leq t(t-1)+(r-t) r,
$$

and by hypothesis $|E(\mathcal{B})| \geq r^{2}-r$. Thus $r^{2}-r \leq t(t-1)+(r-t) r$, yielding $0 \leq(t-r)(t-1)$, which is an absurdity because $1<t<r$. Therefore $|N(S)| \geq|S|$ for all $S \subset X_{1}$, and by Hall's Theorem the lemma follows.

Theorem 8 Let $G$ be $a(\{d, d+1\} ; g)$-cage with odd girth $g \geq 5$, and $d \geq 3$. Then $G$ is edge-superconnected.

Proof: Let us assume that $G$ is a non edge-superconnected $(\{d, d+1\} ; g)$ cage, and we will arrive at a contradiction. To this end, let us take a minimum nontrivial edge-cut $W=\left[W_{0}, W_{1}\right] \subset E(G)$ such that $|W| \leq \delta$. Let $G-W=H_{0} \cup H_{1}$, and let $W_{i} \subset V\left(H_{i}\right)$ be the set of vertices of $H_{i}$ which are incident with some edge in $W, i=0,1$. From Proposition 3 it follows that $\mu_{i}=\max \left\{d\left(x, W_{i}\right): x \in V\left(H_{i}\right)\right\} \geq(g-3) / 2, i=0,1$. Let $x_{i} \in V\left(H_{i}\right) \cap N_{\mu_{i}}\left(W_{i}\right)$. As $G$ is a $(\{d, d+1\} ; g)$-cage, the diameter is at most $\operatorname{diam}(G) \leq g$ by Theorem 2, so we get the following chain of inequalities:

$g \geq \operatorname{diam}(G) \geq d\left(x_{0}, x_{1}\right) \geq d\left(x_{0}, W_{0}\right)+1+d\left(x_{1}, W_{1}\right)=\mu_{0}+1+\mu_{1} \geq g-2$.

If we assume henceforth $\mu_{0} \leq \mu_{1}$ (without loss of generality), then either $(g-3) / 2=\mu_{0} \leq \mu_{1} \leq(g+1) / 2$, or $\mu_{0}=\mu_{1}=(g-1) / 2$. We proceed to study each one of these cases.

In what follows, let $X_{0}, X_{1}$ be two subsets of $V(G)$ such that $\left|X_{0}\right|=$ $\left|X_{1}\right|$. Let $\mathcal{B}_{\Gamma}$ denote the bipartite graph with bipartition $\left(X_{0}, X_{1}\right)$ and $E\left(\mathcal{B}_{\Gamma}\right)=\left\{u_{i} v_{j}: u_{i} \in X_{0}, v_{j} \in X_{1}, d_{\Gamma}\left(u_{i}, v_{j}\right) \geq g-1\right\}$, where $\Gamma$ is a certain subgraph of $G$.

Case (a): $\mu_{0}=(g-3) / 2$. 
Edge-superconnectivity

of semiregular cages with odd girth

C. Balbuena et al.

From Lemma 4 (i), $\left|W_{0}\right|=d=|W|$ so that each vertex of $W_{0}$ is incident to a unique edge of $W$, yielding that every vertex $a \in W_{0}$ has $\operatorname{deg}_{H_{0}}(a) \in\{d-1, d\}$. Also by Lemma 4 (ii), every vertex $x \in N_{(g-3) / 2} \cap$ $V\left(H_{0}\right)$ has $\operatorname{deg}(x)=d$. And by Lemma 4 (iii), for every $a \in W_{0}$ there exists a vertex $x_{0} \in N_{(g-3) / 2} \cap V\left(H_{0}\right)$ such that $N\left(x_{0}\right)=\left\{u_{1}, u_{2}, \ldots, u_{d}\right\}$ and $W_{0}=\left\{a_{1}, a_{2}, \ldots, a_{d}\right\}$, where $a_{1}=a$, in such a way that $d\left(u_{1}, a_{1}\right)=$ $d\left(u_{1}, W_{0}\right)=(g-5) / 2, d\left(u_{j}, W_{0}\right)=d\left(u_{j}, a_{j}\right)=(g-3) / 2$, and by (ii), $\operatorname{deg}\left(u_{j}\right)=d$ for every $j \geq 2$. This implies that $d_{G-x_{0}}\left(u_{1}, a_{j}\right) \geq(g-1) / 2$ for all $j \geq 2$, because the shortest $\left(u_{1}, a_{j}\right)$-path in $G-x_{0}$, the shortest $\left(u_{j}, a_{j}\right)$-path in $G$, and the path $u_{j} x_{0} u_{1}$ in $G$ of length two, form a closed walk containing a cycle. Reasoning analogously, $d_{G-x_{0}}\left(u_{j}, a_{1}\right) \geq(g+1) / 2$ for all $j \geq 2$ and $d_{G-x_{0}}\left(u_{j}, a_{i}\right) \geq(g-1) / 2$ for $j \neq i, j, i \in\{2, \ldots, d\}$. Furthermore, $\left[N_{(g-3) / 2}\left(x_{0}\right) \cap W_{0}, W_{1}\right]=\left\{a_{1} b_{1}\right\}$ for some $b_{1} \in W_{1}$.

Subcase (a.1): $\mu_{1}=(g+1) / 2$.

Let $x_{1} \in V\left(H_{1}\right)$ be any vertex such that $d\left(W_{1}, x_{1}\right)=(g+1) / 2$. Let $X_{0}=$ $\left\{u_{2}, \ldots, u_{d}\right\} \cup\left\{x_{0}\right\}$ and $X_{1}=\left\{v_{1}, v_{2}, \ldots, v_{d}\right\} \subseteq N\left(x_{1}\right)$. As $d\left(u_{i}, W_{0}\right)=$ $(g-3) / 2$ for $i \geq 2$ and $d_{G-x_{1}}\left(W_{1}, N\left(x_{1}\right)\right) \geq(g-1) / 2$, then $d_{G-x_{1}}\left(X_{0}, X_{1}\right) \geq$ $g-1$, so $\left|E\left(\mathcal{B}_{\Gamma}\right)\right|=d^{2}$, where $\Gamma=G-x_{1}$. Clearly $\mathcal{B}_{\Gamma}$ is a complete bipartite graph, so there is a perfect matching $M$ which covers every vertex in $X_{0}$ and if $\operatorname{deg}\left(x_{1}\right)=d$, also covers $N\left(x_{1}\right)$. Hence, in this case the graph $G^{*}=\left(G-\left\{x_{1}\right\}-\left\{x_{0} u_{d}\right\}\right) \cup M$ has girth at least $g$ and the vertices $u_{2}, \ldots, u_{d-1}$ have degree $d+1$ in $G^{*}$ as they had degree $d$ in $G$; for the same reason $x_{0}$ and $u_{d}$ have degree $d$ in $G^{*}$. The remaining vertices have the same degree they had in $G$. As $G^{*}$ is a $\left(\{d, d+1\} ; g^{*}\right)$-graph with girth $g^{*} \geq g$ and $\left|V\left(G^{*}\right)\right|<|V(G)|$, we get a contradiction to the monotonocity Theorem 1 . If $\operatorname{deg}\left(x_{1}\right)=d+1$, since $d_{G^{*}}\left(u_{d}, v_{d+1}\right) \geq g-1$ where $v_{d+1} \in N\left(x_{1}\right) \backslash X_{1}$, we can add the new edge $u_{d} v_{d+1}$ to $G^{*}$ without decreasing the girth. Then $G^{*} \cup\left\{u_{d} v_{d+1}\right\}$ gives us again a contradiction.

Subcase (a.2): $\mu_{1}=(g-3) / 2$.

By Lemma 4 , given $b_{1} \in W_{1}$ there exists $x_{1} \in V\left(H_{1}\right) \cap N_{(g-3) / 2}\left(W_{1}\right)$ of $\operatorname{deg}\left(x_{1}\right)=d$ such that $N\left(x_{1}\right)=\left\{v_{1}, v_{2}, \ldots, v_{d}\right\}, W_{1}=\left\{b_{1}, b_{2}, \ldots, b_{d}\right\}$ and each vertex of $W_{1}$ is incident to a unique edge of $W$, hence $W=$ $\left\{a_{1} b_{1}, a_{2} b_{2}, \ldots, a_{d} b_{d}\right\}$. Also, $d\left(b_{1}, v_{1}\right)=d\left(W_{1}, v_{1}\right)=(g-5) / 2$, and $d\left(W_{1}, v_{j}\right)=$ $d\left(b_{j}, v_{j}\right)=(g-3) / 2$ for every $j \geq 2$ and besides $\operatorname{deg}\left(v_{j}\right)=d$. Then $d\left(x_{0}, x_{1}\right)=d\left(x_{0}, a_{1}\right)+1+d\left(b_{1}, x_{1}\right)=g-2$, and if $g=5$ it is easy to see that the shortest $\left(x_{0}, x_{1}\right)$-path of length three is unique, clearly $x_{0} a_{1} b_{1} x_{1}$. 
Edge-superconnectivity

of semiregular cages with odd girth

C. Balbuena et al.

Now let $\Gamma=G-\left\{x_{0}, x_{1}\right\}$. We have

$$
\begin{gathered}
d_{\Gamma}\left(u_{1}, N\left(x_{1}\right)-v_{1}\right)=\min \left\{d_{\Gamma}\left(u_{1}, a_{1}\right)+1+d_{\Gamma}\left(b_{1}, N\left(x_{1}\right)-v_{1}\right) ;\right. \\
\left.d_{\Gamma}\left(u_{1}, a_{j}\right)+1+d_{\Gamma}\left(b_{j}, N\left(x_{1}\right)-v_{1}\right), j \geq 2\right\} \\
\geq \min \left\{\frac{g-5}{2}+1+\frac{g+1}{2} ; \frac{g-1}{2}+1+\frac{g-3}{2}\right\}=g-1,
\end{gathered}
$$

since $d_{\Gamma}\left(b_{1}, v_{j}\right) \geq(g+1) / 2$ for all $j \geq 2$, because the shortest $\left(b_{1}, v_{j}\right)$-path in $\Gamma$, the shortest $\left(b_{1}, v_{1}\right)$-path in $\Gamma$, and the path $v_{j} x_{1} v_{1}$ in $G$ of length two, form a closed walk containing a cycle. Reasoning in the same way, it follows for all $j \geq 2$ that

$$
\begin{aligned}
& d_{\Gamma}\left(u_{j}, N\left(x_{1}\right)-v_{j}\right)= \\
& =\min \left\{d_{\Gamma}\left(u_{j}, a_{j}\right)+1+d_{\Gamma}\left(b_{j}, N\left(x_{1}\right)-v_{j}\right) ; d_{\Gamma}\left(u_{j}, a_{h}\right)+1\right. \\
& \left.\quad+d_{\Gamma}\left(b_{h}, N\left(x_{1}\right)-v_{j}\right), h \neq j\right\} \\
& \quad \min \left\{\begin{array}{l}
\left\{\frac{g-3}{2}+1+\frac{g-1}{2} ; \frac{g-1}{2}+1+\frac{g-3}{2}\right\} \text { if } h \geq 2, h \neq j \\
\left\{\frac{g-3}{2}+1+\frac{g-1}{2} ; \frac{g+1}{2}+1+\frac{g-5}{2}\right\} \text { if } h=1
\end{array}\right\} \\
& =g-1 .
\end{aligned}
$$

Analogously, $d_{\Gamma}\left(N\left(x_{0}\right)-u_{1}, v_{1}\right) \geq g-1$ and $d_{\Gamma}\left(N\left(x_{0}\right)-u_{j}, v_{j}\right) \geq g-1$ for all $j \geq 2$. Let $X_{0}=N\left(x_{0}\right)$ and $X_{1}=N\left(x_{1}\right)$. The bipartite graph $\mathcal{B}_{\Gamma}=\left(X_{0}, X_{1}\right)$ has $\left|E\left(\mathcal{B}_{\Gamma}\right)\right|=d^{2}-d$ and $\operatorname{deg}_{\mathcal{B}_{\Gamma}}(w) \geq 1$ for all $w \in X_{0} \cup X_{1}$. From Lemma 7, there is a perfect matching $M$ between $X_{0}=N\left(x_{0}\right)$ and $X_{1}=N\left(x_{1}\right)$. Hence $G^{*}=\left(G-\left\{x_{0}, x_{1}\right\}\right) \cup M$ is a $\left(\{d, d+1\} ; g^{*}\right)$-graph (because every vertex in $G^{*}$ has the same degree it had in $G$ and the removed vertices $x_{0}, x_{1}$ had degree $d$, as well as the vertices $u_{j}, v_{k}$ for every $j, k \geq 2$ ) with $g^{*} \geq g$ and $\left|V\left(G^{*}\right)\right| \leq|V(G)|$, which contradicts the monotonocity Theorem 1 , and we are done.

Subcase (a.3): $\mu_{1}=(g-1) / 2$. In this case we distinguish two other possible subcases.

Subcase (a.3.1): There exists $x_{1} \in V\left(H_{1}\right) \cap N_{(g-1) / 2}\left(W_{1}\right)$ such that $d(b, v) \leq(g-1) / 2$ for all $b \in W_{1}$ and for all $v \in N\left(x_{1}\right)$.

Then, every $b \in W_{1}$ has $\operatorname{deg}_{H_{1}}(b)=\operatorname{deg}\left(x_{1}\right) \in\{d, d+1\}$ because $d(b, v) \leq(g-1) / 2$ and $\left|N_{(g-3) / 2}(v) \cap N(b)\right| \leq 1$ for all $v \in N\left(x_{1}\right)$ (otherwise 
Edge-superconnectivity

of semiregular cages with odd girth

C. Balbuena et al.

cycles of length less than $g$ appear). Hence $\operatorname{deg}\left(x_{1}\right)=d$ and $\operatorname{deg}(b)=$ $d+1$ for all $b \in W_{1}$. Thus $N\left(x_{1}\right)=\left\{v_{1}, \ldots, v_{d}\right\}$ and $W=\left[W_{0}, W_{1}\right]$ is a matching, i.e., $W=\left\{a_{1} b_{1}, \ldots, a_{d} b_{d}\right\}$. Therefore the subgraph $H_{1}$ gives a contradiction unless $H_{1}$ is $d$-regular. In this case let us consider the graph $\hat{G}=\left(G-x_{1}-W\right) \cup\left\{a_{1} v_{1}, \ldots, a_{d} v_{d}\right\}$ which clearly has girth at least $g$. Moreover $\operatorname{deg}_{\hat{G}}\left(b_{i}\right)=\operatorname{deg}\left(b_{i}\right)-1=d$ and every vertex different from $b_{i}$ has the same degree it had in $G$. Thus we may suppose that $\hat{G}$ is $d$ regular because otherwise $\hat{G}$ would be a $\left(\{d, d+1\} ; g^{*}\right)$-graph with girth $g^{*} \geq g$ and smaller than $G$, a contradiction. Moreover, we may assume that $d_{H_{1}}\left(b_{1}, v_{1}\right)=(g-3) / 2$ and $d_{H_{1}}\left(b_{1}, N\left(x_{1}\right)-v_{1}\right)=(g-1) / 2$. Thus we have

$$
\begin{aligned}
d_{\hat{G}}\left(b_{1}, u_{2}\right) \geq & \min \left\{d_{H_{1}}\left(b_{1}, v_{2}\right)+\left|\left\{v_{2} a_{2}\right\}\right|\right. \\
& \left.+d_{H_{0}}\left(a_{2}, u_{2}\right) ; d_{H_{1}}\left(b_{1}, v_{1}\right)+\left|\left\{v_{1} a_{1}\right\}\right|+d_{H_{0}}\left(a_{1}, u_{2}\right)\right\} \\
\geq & \min \left\{\frac{g-1}{2}+1+\frac{g-3}{2} ; \frac{g-3}{2}+1+\frac{g+1}{2}\right\} \\
= & g-1,
\end{aligned}
$$

which implies that we can add to $\hat{G}$ the edge $u_{2} b_{1}$ to obtain a graph without decreasing the girth $g$. As this new graph is smaller than $G$ and has degrees $\{d, d+1\}$ we get a contradiction to the monotonicity Theorem 1 , and we are done.

Subcase (a.3.2): For all $z \in V\left(H_{1}\right) \cap N_{(g-1) / 2}\left(W_{1}\right)$ there exists $v \in$ $N\left(x_{1}\right)$ and $b \in W_{1}$ such that $d(b, v) \geq(g+1) / 2$.

Let $x_{1} \in V\left(H_{1}\right) \cap N_{(g-1) / 2}\left(W_{1}\right), v_{1} \in N\left(x_{1}\right)$ and $b^{*} \in W_{1}$ be such that $d\left(b^{*}, v_{1}\right) \geq(g+1) / 2$. By Lemma 4 , there exists a unique edge $a^{*} b^{*} \in W$ to which the vertex $a^{*} \in W_{0}$ is incident, and there exists a vertex $x^{*} \in V\left(H_{0}\right)$ of $\operatorname{deg}\left(x^{*}\right)=d$ such that $d\left(x^{*}, W_{0}\right)=d\left(x^{*}, a^{*}\right)=$ $(g-3) / 2$ and $N_{(g-3) / 2}\left(x^{*}\right) \cap W_{0}=\left\{a^{*}\right\}$. Further, $N\left(x^{*}\right)$ can be labeled as $\left\{z_{1}, z_{2}, \ldots, z_{d}\right\}$, and $W_{0}$ can be labeled as $\left\{a_{1}, a_{2}, \ldots, a_{d}\right\}$, where $a_{1}=a^{*}$, so that $N_{(g-5) / 2}\left(z_{1}\right) \cap W_{i}=\left\{a_{1}\right\}, N_{(g-3) / 2}\left(z_{k}\right) \cap W_{i}=\left\{a_{k}\right\}$ and $\operatorname{deg}\left(z_{k}\right)=d$ for every $k>1$. Furthermore, $\left[N_{(g-3) / 2}\left(x^{*}\right) \cap W_{0}, W_{1}\right]=\left\{a_{1} b^{*}\right\}$

Let $\Gamma=G-\left\{x^{*}, x_{1}\right\}$. We obtain

$$
\begin{aligned}
& d_{\Gamma}\left(z_{1}, v_{1}\right) \\
& =\min \left\{d_{\Gamma}\left(z_{1}, a_{1}\right)+1+d_{\Gamma}\left(b^{*}, v_{1}\right) ; d_{\Gamma}\left(z_{1}, a_{j}\right)+1+d_{\Gamma}\left(b^{\prime}, v_{1}\right), j \geq 2, a_{j} b^{\prime} \in W\right\} \\
& \geq \min \left\{\frac{g-5}{2}+1+\frac{g+1}{2} ; \frac{g-1}{2}+1+\frac{g-3}{2}\right\}=g-1 .
\end{aligned}
$$


Edge-superconnectivity

of semiregular cages with odd girth

C. Balbuena et al.

Moreover, $d_{H_{0}}\left(z_{k}, W_{0}\right)=(g-3) / 2$ for all $z_{k} \in N\left(x^{*}\right)-z_{1}$ and for $k>1$ there exists a unique vertex say $b_{k} \in W_{1}$ for which $a_{k} b_{k} \in W$. As for each $b \in W_{1},\left|N_{(g-3) / 2}(b) \cap N\left(x_{1}\right)\right| \leq 1$ (otherwise cycles of length less than $g$ appear) we may denote by $v_{k}$ the vertex in $N\left(x_{1}\right)-v_{1}$ such that $d\left(b_{k}, v_{k}\right)=(g-3) / 2$, if any. Thus we obtain

$$
\begin{aligned}
& d_{\Gamma}\left(z_{k}, N\left(x_{1}\right) \backslash\left\{v_{1}, v_{k}\right\}\right)=d\left(z_{k}, a_{k}\right)+1+d\left(b_{k}, N\left(x_{1}\right) \backslash\left\{v_{1}, v_{k}\right\}\right) \\
& \geq \frac{g-3}{2}+1+\frac{g-1}{2}=g-1 .
\end{aligned}
$$

Let us consider $X_{0}=N\left(x^{*}\right)-z_{1}$ and $X_{1} \subseteq N\left(x_{1}\right)-v_{1}$, with $\left|X_{1}\right|=d-1$. It is clear that $\left|\operatorname{deg}_{\mathcal{B}_{\Gamma}}\left(z_{k}\right)\right| \geq d-2 \geq 1$ for all $z_{k} \in N\left(x^{*}\right)-u_{1}$ yielding $\left|E\left(\mathcal{B}_{\Gamma}\right)\right| \geq(d-2)(d-1)=(d-1)^{2}-(d-1)$.

First, suppose that $\left|\operatorname{deg}_{\mathcal{B}_{\Gamma}}(v)\right| \geq 1$ for all $v \in N\left(x_{1}\right)-v_{1}$. From Lemma 7 , there is a matching $M$ which covers every vertex in $N\left(x^{*}\right)-z_{1}$ and every vertex in $N\left(x_{1}\right)-v_{1}$ if $\operatorname{deg}\left(x_{1}\right)=d$. In this case $G^{*}=\left(G-\left\{x^{*}, x_{1}\right\}\right) \cup$ $M \cup\left\{z_{1} v_{1}\right\}$ is a graph with girth $g^{*} \geq g$ and smaller than $G$ whose vertices have the same degree they had in $G$; thus $G^{*}$ is a $\left(\{d, d+1\} ; g^{*}\right)$-graph and we are done. Thus suppose that $\operatorname{deg}\left(x_{1}\right)=d+1$ and that after adding the matching $M \cup\left\{z_{1} v_{1}\right\}$ to $G-\left\{x^{*}, x_{1}\right\}$ the vertex $v_{d+1} \in\left(N\left(x_{1}\right)-v_{1}\right) \backslash X_{1}$ remains of degree $d-1$. By Lemma 4 every $z_{k}, k>1$, has degree $d$ in $G$, and we have proved that $d\left(z_{k}, N\left(x_{1}\right) \backslash\left\{v_{1}, v_{k}\right\}\right) \geq g-1$. Then we add one extra edge $z_{k} v_{d+1}$ to $G^{*}$ obtaining a new $\left(\{d, d+1\} ; g^{*}\right)$-graph with $g^{*} \geq g$ and smaller than $G$, a contradiction to the monotonicity Theorem 1, so we are done.

Therefore we must suppose that there exists $v_{2} \in N\left(x_{1}\right)-v_{1}$ such that $\left|\operatorname{deg}_{\mathcal{B}_{\Gamma}}\left(v_{2}\right)\right|=0$. This implies that $d\left(v_{2}, b\right)=(g-3) / 2$ for all $b \in W_{1}-b^{*}$, hence $d\left(v, W_{1}-b^{*}\right)=(g-1) / 2$ for all $v \in N\left(x_{1}\right)-v_{2}$. First suppose that $d\left(v_{2}, b^{*}\right) \geq(g+1) / 2 ;$ then $d_{\Gamma}\left(z_{1}, v_{2}\right) \geq g-1, d_{\Gamma}\left(z_{k}, N\left(x_{1}\right)-v_{2}\right)=g-1$ for all $k \geq 2$, thus we consider the set $X_{1} \subseteq N\left(x_{1}\right)-v_{2}$ with $\left|X_{1}\right|=d-1$. It is clear that $\left|\operatorname{deg}_{\mathcal{B}_{\Gamma}}(w)\right| \geq d-1$ for all $w \in X_{0} \cup X_{1}$. Using Lemma 7 and reasoning as before we get a contradiction. Therefore we must suppose that $d\left(v_{2}, b^{*}\right) \leq(g-1) / 2$. Since $N\left(x_{1}\right)-v_{2} \subseteq N_{(g-1) / 2}\left(W_{1}\right) \cap V\left(H_{1}\right)$ we have by hypothesis that for all $v \in N\left(x_{1}\right)-v_{2}$ there exists $\hat{v}_{1} \in N(v)$ and $\hat{b}^{*} \in W_{1}$ such that $d\left(\hat{b}^{*}, \hat{v}_{1}\right) \geq(g+1) / 2$. As the behavior of any $v \in N\left(x_{1}\right)-v_{2}$ is the same as vertex $x_{1}$, reasoning as before we get a contradiction unless for all $v \in N\left(x_{1}\right)-v_{2}$ there exists $\hat{v}_{2} \in N(v)-\hat{v}_{1}$ such that $\left|\operatorname{deg}_{\mathcal{B}_{\hat{\Gamma}}}\left(\hat{v}_{2}\right)\right|=0$ satisfying $d\left(\hat{v}_{2}, b\right)=(g-3) / 2$ for all $b \in W_{1}-\hat{b}^{*}$ and $d\left(\hat{v}_{2}, \hat{b}^{*}\right) \leq(g-1) / 2$. Therefore we conclude that every vertex $b \in W_{1}$ has 
Edge-superconnectivity

of semiregular cages with odd girth

C. Balbuena et al.

$\operatorname{deg}_{H_{1}}(b)=\operatorname{deg}\left(x_{1}\right) \in\{d, d+1\}$. Now considering the same graph as in Subcase (a.3.1) we get a contradiction.

Case (b): $\mu_{0}=\mu_{1}=(g-1) / 2$.

Let $x_{0} \in V\left(H_{0}\right)$ and $x_{1} \in V\left(H_{1}\right)$ satisfy $d\left(x_{i}, W_{i}\right)=(g-1) / 2, i=0,1$.

First of all note that there must exist a vertex in $N\left(x_{0}\right)$ of degree $d$, otherwise $G-x_{0}$ would be either a $\{d, d+1\}$-graph or a $d$-regular graph. In the former case we get a contradiction because $G-x_{0}$ is smaller than $G$ and has girth at least $g$. And in the latter case we consider the graph $\left(G-x_{0}\right) \cup\left\{u_{i} x_{1}\right\}$ with $u_{i} \in N\left(x_{0}\right)$, which gives again a contradiction. Similarly, note that there must exist a vertex in $N\left(x_{1}\right)$ of degree $d$.

Suppose that $\operatorname{deg}\left(x_{0}\right)=\operatorname{deg}\left(x_{1}\right)=r$ with $r \in\{d, d+1\}$. Let $X_{0}=$ $N\left(x_{0}\right), X_{1}=N\left(x_{1}\right)$ and $\Gamma=G-\left\{x_{0}, x_{1}\right\}$. Define $A=\left\{u_{i} v_{j}: u_{i} \in X_{0}, v_{j} \in\right.$ $\left.X_{1}, d_{\Gamma}\left(u_{i}, v_{j}\right) \leq g-2\right\}$ and consider $\mathcal{B}_{\Gamma}=K_{\left|X_{0}\right|,\left|X_{1}\right|}-A$. Note that every $\left(u_{i}, v_{j}\right)$-path in $G$ goes through an edge of $W$. Therefore every edge in $W$ gives rise to at most one element in $A$, otherwise $G$ would contain a cycle of length at most $2(g-3) / 2+2=g-1$. Hence $|A| \leq|W| \leq d$ and $\left|E\left(\mathcal{B}_{\Gamma}\right)\right|=\left|K_{r, r}\right|-|A| \geq r^{2}-d$.

If $r=d+1$ then $\left|E\left(\mathcal{B}_{\Gamma}\right)\right| \geq(d+1)^{2}-d=d^{2}+d+1$ and by Lemma 6 , the graph $\mathcal{B}_{\Gamma}$ contains a perfect matching $M$. Therefore the graph $G^{\prime}=$ $G-\left\{x_{0}, x_{1}\right\} \cup M$ has fewer vertices than $G$ and girth at least $g$ producing a contradiction unless $G^{\prime}$ is regular of degree $d$. In this case we consider the graph $G^{\prime \prime}=G^{\prime} \cup\{u v\}$ where $u \in N\left(x_{0}\right)$ is such that $d\left(u, W_{0}\right)=(g-1) / 2$ (such a vertex must exist because $\operatorname{deg}\left(x_{0}\right)=d+1$ and $\left|W_{1}\right| \leq d$ ) and $v \in N\left(x_{1}\right)$ such that $u v \notin M$. As $G^{\prime \prime}$ is a $(\{d, d+1\} ; g)$-graph with fewer vertices than $G$ and girth $g$ a contradiction is again obtained.

Suppose $r=d$. If $\operatorname{deg}_{\mathcal{B}_{\Gamma}}(z) \geq 1$ for all $z \in \mathcal{B}_{\Gamma}$, then by Lemma 7 there exists a perfect matching $M$ between $X_{0}$ and $X_{1}$; reasoning as before we obtain again a contradiction. Hence, we may assume that $\operatorname{deg}_{\mathcal{B}_{\Gamma}}\left(u_{1}\right)=0$ for some $u_{1} \in X_{0}$. This implies that $d_{\Gamma}\left(u_{1}, v_{j}\right)=g-2$ for all $v_{j} \in N\left(x_{1}\right)$, or equivalently $d_{\Gamma}\left(v_{j}, W_{1}\right)=(g-3) / 2$ for all $v_{j} \in N\left(x_{1}\right)$. From this, and because $g \geq 5$, we get $\left|W_{1}\right| \geq\left|N\left(x_{1}\right)\right|=d$, yielding $\left|W_{1}\right|=d$ (since $d=$ $\left.|W| \geq\left|W_{1}\right|\right)$, and also $N_{(g-3) / 2}\left(v_{j}\right) \cap W_{1}=\left\{b_{j}\right\}$ for all $v_{j} \in N\left(x_{1}\right)$. That is, $\left|N\left(b_{j}\right) \cap W_{0}\right|=1$ for every $b_{j} \in W_{1}$. Also we have $N_{(g-1) / 2}\left(u_{1}\right) \cap W_{1}=W_{1}$, hence $N_{(g-3) / 2}\left(u_{1}\right) \cap W_{0}=W_{0}$ and thus $d\left(u_{i}, W_{0}\right)=(g-1) / 2$ for $i \geq 2$. 
Edge-superconnectivity

of semiregular cages with odd girth

C. Balbuena et al.

Let $u_{k} \in N\left(x_{0}\right), k \geq 2$, define $\Gamma_{k}=G-\left\{u_{k}, x_{1}\right\}$ and consider the sets

$$
\begin{aligned}
X_{k} & = \begin{cases}N\left(u_{k}\right) & \text { if } \operatorname{deg}\left(u_{k}\right)=d ; \\
N\left(u_{k}\right)-x_{0} & \text { if } \operatorname{deg}\left(u_{k}\right)=d+1 ;\end{cases} \\
X_{1} & =N\left(x_{1}\right) ; \\
A_{k} & =\left\{z_{i} v_{j}: z_{i} \in X_{k}, v_{j} \in X_{1}, d_{\Gamma_{k}}\left(z_{i}, v_{j}\right) \leq g-2\right\} .
\end{aligned}
$$

Let $\mathcal{B}_{\Gamma_{k}}=K_{\left|X_{k}\right|,\left|X_{1}\right|}-A_{k}$.

If $\operatorname{deg}_{\mathcal{B}_{\Gamma_{k}}}(z) \geq 1$ for all $z \in X_{k}$, we get a perfect matching $M$ between $X_{k}$ and $N\left(x_{1}\right)$ by Lemma 7 ; if $\operatorname{deg}\left(u_{k}\right)=d$ the graph $\Gamma_{k} \cup M$ yields a contradiction; if $\operatorname{deg}\left(u_{k}\right)=d+1$ the graph $\Gamma_{k} \cup M \cup\left\{x_{0} v_{j}\right\}$, where $v_{j}$ is a vertex of $N\left(x_{1}\right)$ with degree $d$, yields again a contradiction. Therefore we can suppose that for every $u_{k} \in N\left(x_{0}\right)-u_{1}$ there exists $\hat{z}_{k} \in N\left(u_{k}\right)$ such that $d_{\Gamma_{k}}\left(\hat{z}_{k}, v_{j}\right)=g-2$ for all $v_{j} \in N\left(x_{1}\right)$. Hence, $N_{(g-3) / 2}\left(\hat{z}_{k}\right) \cap W_{0}=W_{0}$, that is $d_{\Gamma_{k}}\left(\hat{z}_{k}, a_{j}\right)=(g-3) / 2$ for each $a_{j} \in W_{0}$. Therefore $\operatorname{deg}_{H_{0}}\left(a_{j}\right)=d$, $\operatorname{deg}\left(a_{j}\right)=d+1$ and $\left[W_{0}, W_{1}\right]$ is a matching (recall that $\left|N\left(b_{j}\right) \cap W_{0}\right|=1$ for every $\left.b_{j} \in W_{1}\right)$. We can now use the same graph $\hat{G}=\left(G-\left\{x_{0}\right\}-W\right) \cup$ $\left\{b_{1} u_{1}, \ldots, b_{d} u_{d}\right\}$ as used in Case (a.3.2), arriving again at a contradiction.

The only remaining case occurs when $x_{0}$ and $x_{1}$ have different degrees. Let us suppose $\operatorname{deg}\left(x_{0}\right)=d$ and $\operatorname{deg}\left(x_{1}\right)=d+1$. As $\operatorname{deg}\left(x_{1}\right)=d+1>\left|W_{1}\right|$, there exists, say $v_{d+1} \in N\left(x_{1}\right)$, such that $d\left(v_{d+1}, W_{1}\right)=(g-1) / 2$. We proceed as before, with the sets $X_{0}=N\left(x_{0}\right)$ and $X_{1}=N\left(x_{1}\right)-v_{d+1}$, finding a graph $G^{\prime}$ with fewer vertices and the same girth and degrees as $G$, except for the vertex $v_{d+1}$. Recall that there must exist a vertex $y \in N\left(x_{0}\right)$ such that $\operatorname{deg}(y)=d$. Then we construct the graph $G^{*}=G^{\prime} \cup\left\{y v_{d+1}\right\}$, which is a new $\{d, d+1\}$-graph with girth $g$, arriving at a contradiction. This ends the proof of the theorem.

\section{Acknowledgement}

This research was supported by the Ministry of Education and Science, Spain, and the European Regional Development Fund (ERDF) under project MTM2008-06620-C03-02/MTM; also by Catalonian government 2009 SGR 1298. The author J. Salas has been supported by CONACYT. 
Edge-superconnectivity

of semiregular cages with odd girth

C. Balbuena et al.

\section{References}

[1] M.C. Balbuena, A. Carmona, J. Fàbrega, and M.A. Fiol. On the order and size of $s$-geodetic digraphs with given connectivity. Discrete Math. 174:19-27, 1997.

[2] C. Balbuena, P. García-Vázquez, and X. Marcote. Sufficient conditions for $\lambda^{\prime}$-optimality in graphs with girth $g$. J. Graph Theory 52:73-86, 2006 .

[3] C. Balbuena, D. González-Moreno, and X. Marcote. On the connectivity of semiregular cages. Networks 56(8):81-88, 2010.

[4] C. Balbuena and X. Marcote. Monotonicity of the order of $(D ; g)$ cages. Submitted.

[5] C. Balbuena and X. Marcote. Diameter and connectivity of $(D ; g)$ cages. International Journal of Computer Math., accepted.

[6] F.T. Boesch. Synthesis of reliable networks-A survey. IEEE Trans Reliability, 35:240-246, 1986.

[7] F.T. Boesch and R. Tindell. Circulants and their connectivities. J. Graph Theory 8:487-499, 1984.

[8] G. Chartrand, R.J. Gould, and S.F. Kapoor. Graphs with prescribed degree set and girth. Period. Math Hungar. 6:261-266, 1981.

[9] G. Chartrand and L. Lesniak. Graphs and digraphs, 3rd ed. Chapman and Hall, London, 1996.

[10] P. Erdös and H. Sachs. Reguläre Graphen gegebener Taillenweite mit minimaler Knotenzahl. Wiss. Z. Martin-Luther-Univ. HalleWittenberg Math.-Natur. Reihe, 12:251-257, 1963.

[11] H. Fu, K. Huang, and C. Rodger. Connectivity of cages. J. Graph Theory, 24:187-191, 1997.

[12] P. Hall. On representatives of subsets. J. London Math. Soc., 10:26-30, 1935. 
Edge-superconnectivity

of semiregular cages with odd girth

C. Balbuena et al.

[13] M. Imase, T. Soneoka, and K. Okada. Connectivity of regular directed graphs with small diameter. IEEE Trans Comput., C-34:267$273,1985$.

[14] T. Jiang. Short even cycles in cages with odd girth. Ars Combin., 59:165-169, 2001.

[15] T. Jiang and D. Mubayi. Connectivity and separating sets of cages. J. Graph Theory, 29:35-44, 1998.

[16] Y. Lin, C. Balbuena, X. Marcote, and M. Miller. On the connectivity of $(k, g)$-cages of even girth. Discrete Math., 308:3249-3256, 2008.

[17] Y. Lin, M. Miller, and C. Balbuena. Improved lower bound for the vertex connectivity of $(\delta ; g)$-cages. Discrete Math., 299:162-171, 2005.

[18] Y. Lin, M. Miller, C. Balbuena, and X. Marcote. All $(k ; g)$-cages are edge-superconnected. Networks, 47:102-110, 2006.

[19] X. Marcote and C. Balbuena. Edge-superconnectivity of cages. Networks, 43:54-59, 2004.

[20] X. Marcote, C. Balbuena, I. Pelayo, and J. Fbrega. $(\delta, g)$-cages with $g \geq 10$ are 4-connected. Discrete Math., 301:124-136, 2005. 
